Article

\title{
Water Transport and Removal in PEMFC Gas Flow Channel with Various Water Droplet Locations and Channel Surface Wettability
}

\author{
Yanzhou Qin * (D), Xuefeng Wang, Rouxian Chen and Xiang Shangguan \\ State Key Laboratory of Engines, Tianjin University, 300072 Tianjin, China; 2016201278@tju.edu.cn (X.W.); \\ tjucrx@163.com (R.C.); 15620493892@163.com (X.S.) \\ * Correspondence: qinyanzhou@tju.edu.cn \\ Received: 6 February 2018; Accepted: 4 April 2018; Published: 10 April 2018

\begin{abstract}
Water transport and removal in the proton exchange membrane fuel cell (PEMFC) is critically important to fuel cell performance, stability, and durability. Water emerging locations on the membrane-electrode assembly (MEA) surface and the channel surface wettability significantly influence the water transport and removal in PEMFC. In most simulations of water transport and removal in the PEMFC flow channel, liquid water is usually introduced at the center of the MEA surface, which is fortuitous, since water droplet can emerge randomly on the MEA surface in PEMFC. In addition, the commonly used no-slip wall boundary condition greatly confines the water sliding features on hydrophobic MEA/channel surfaces, degrading the simulation accuracy. In this study, water droplet is introduced with various locations along the channel width direction on the MEA surface, and water transport and removal is investigated numerically using an improved model incorporating the sliding flow property by using the shear wall boundary condition. It is found that the water droplet can be driven to the channel sidewall by aerodynamics when the initial water location deviates from the MEA center to a certain amount, forming the water corner flow in the flow channel. The channel surface wettability on the water transport is also studied and is shown to have a significant impact on the water corner flow in the flow channel.
\end{abstract}

Keywords: proton exchange membrane fuel cell; water management; water transport; water removal; water droplet location; water corner flow

\section{Introduction}

Proton exchange membrane fuel cell (PEMFC) is a promising clean power source for future applications, especially for the automobiles, due to its low emission, high efficiency, and high current density. Water management is one of the critical issues in PEMFC. In PEMFC, sufficient gas humidification is indispensable to the effective hydration of the membrane for good proton conductivity; on the other hand, high gas humidity and continuous water production in the cathode catalyst layer frequently leads to flooding of the cathode electrode and gas flow channel, causing the reactant gas depletion due to the transport barrier. Effective water management therefore requires a subtle water balance to maintain the delicate equilibrium between membrane dehydration and water flooding of the electrode and flow channel, minimizing both of their negative effects on the fuel cell performance. Due to the complexity of water transport and the removal mechanisms in PEMFC, water management remains a major concern and challenge in the application of PEMFC, which still requires much work to be done on the basic understanding of water transport and removal in PEMFC. Water management in PEMFC can be investigated both numerically [1,2] and experimentally [3]. Water transport and removal in both the gas flow channel [4,5] and porous electrode [6] are important 
to fuel cell performance. Water flooding can be diagnosed by monitoring the voltage-time curve [7], humidity [8], and pressure drop of PEMFC [9-11].

Water is produced by the electrochemical reaction in the cathode catalyst layer. Liquid water is formed and accumulated in the porous electrode when water vapor is condensed, and it is mainly removed by the air flow in the cathode flow channel after emerging from the membrane-electrode assembly (MEA) surface. For water management in PEMFC, it is essential to understand the water removal process from the MEA surface and then control the water removal process to minimize the barrier for reactant gas transport and the pressure drop or the parasitic power of gas supply. Water removal from the MEA surface can be affected by multiple parameters, such as the gas velocity, the channel design, the water droplet size and emerging location on MEA surface, and the surface wettability represented by the contact angle.

So far, a number of studies have analyzed the liquid water transport and removal process from the MEA surface. Liquid water detachment on the MEA surface was experimentally and theoretically investigated by Zhang et al. [12]. Their results showed that the water detachment diameter decreased with the air velocity. At high air velocity, small water droplets were easily detached from the MEA surface by strong air shear force, while at low air velocity, water droplets grew to the channel dimension size and accumulated, forming the corner flow, annular flow, and slug flow, and water in this situation was mainly removed by the channel surface wetting effect. Chen et al. [13] further found that water removal was enhanced by increasing the channel length, the air velocity and the MEA surface hydrophobicity, and/or the decreasing the channel height and the surface contact angle hysteresis. Kumbur et al. [14] pointed out that increasing the Polytetrafluoroethylene (PTFE) content of the diffusion media facilitated water removal from the MEA surface considerably at high air velocity, and the PTFE content impact was small at low air velocity. Theodorakakos et al. [15] showed that the water deformation represented by the contact angle hysteresis was smaller for the larger water droplet and surface static contact angle, and water detachment became easier when the surface tension between the water and air was decreased by increasing the temperature. Xie et al. [16] built the criteria of the critical parameters for droplet detachment on the inclined surface and the subsequent flow mode after droplet detachment.

Owing to the improvement of computational capability, detailed liquid water transport and removal is available through two-phase numerical simulation with interface tracking technique, such as the VOF (volume of fluid) method. Quan et al. [17] investigated water transport through a U-turn in a serpentine flow channel using the VOF method and revealed that the U-turn had an important influence on water transport and removal in the flow channel, and the secondary flow induced by the air-water interaction at the bend area strongly affected the water behaviors either in the bend or after the bend area. Jiao et al. [18] studied water transport in a fuel cell stack with serpentine flow channel layout and pointed out that water was not evenly distributed in the flow channel, which could cause the reactant gas distribution and fuel cell performance deviation among fuel cells. Water transport and removal in the flow channel with various channel cross section shapes was analyzed by Zhu et al. [19] and in the GDL with various designs by Park et al. [20] and Yin et al. [21]. Using the same approach, Cai et al. [22] investigated the channel/MEA surface wettability (static contact angle) on water transport and found that water droplet was transported faster on a more hydrophobic surface. Water could be removed by the channel sidewall wetting effect if the channel surface was hydrophilic, thus forming water corner flow in the channel. Qin et al. [23-25] enhanced the water removal from the MEA surface by adding a hydrophilic needle and plate in the flow channel, and the water corner flow in the hydrophilic channel surface was also identified in their studies.

In most numerical studies, the water droplet is usually considered and introduced at the center of the MEA surface and only transported along the central line of the MEA surface in the flow channel, which is fortuitous, since the water droplet can emerge randomly at locations along the channel width direction on the MEA surface. Water transport and removal may change significantly at different initial water locations in the flow channel due to the change in the flow field surrounding the water droplet. 
Furthermore, the water sliding flow on hydrophobic MEA/channel surfaces has been recognized by researchers. However, the no-slip wall boundary condition is still commonly used in the numerical simulation, which is inconsistent with the water sliding phenomena in PEMFC and reduces the simulation accuracy [26-28]. The sliding angle, which is defined as the critical angle when the liquid droplet begins to slide on an inclined solid surface under the gravity force, as illustrated in Figure 1, is usually employed to measure the resistance of water moving and sliding on the solid surface in the experimental study [29], and it also needs to be introduced in the numerical simulation of water flow in PEMFC. It is worth mentioning that although the sliding angle is defined by the gravity force, it essentially represents the critical resistant force for the droplet motion, regardless of the driving force format.

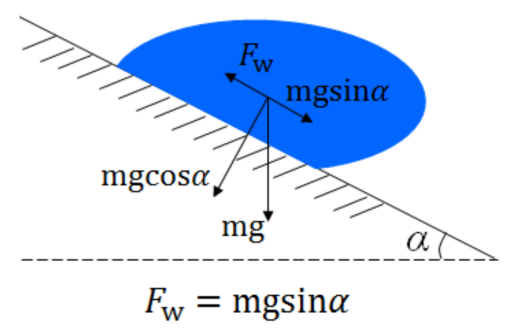

Figure 1. Illustrations of the sliding angle and the wall adhesion force. $\alpha$ is the sliding angle and $F_{\mathrm{w}}$ is the wall adhesion force.

In this study, water transport and removal in PEMFC gas flow channel will be investigated numerically using the VOF method incorporating the water sliding property, which is measured by the sliding angle. Various water droplet locations will be considered and investigated on water transport and removal in the PEMFC flow channel. The channel surface wettability (including both the contact angle and sliding angle) on water removal from the MEA surface will also be discussed.

\section{Model Formulation}

\subsection{Computational Domain and Assumptions}

A single straight cathode flow channel and its boundaries constitute the computational domain, as shown in Figure 2. The channel has a length of $30 \mathrm{~mm}$ and a typical square cross section of $1 \mathrm{~mm} \times 1 \mathrm{~mm}$. The liquid water droplet is initially introduced on the MEA surface, since it emerges from the MEA surface to the gas flow channel. The MEA surface and the channel surface wettability is characterized by the contact angle and sliding angle of water on the surfaces. The air is considered as the gas flow in the channel, and the phase change between the two air-water phases is neglected considering the fast water transport in the channel. It is assumed that the air-water two-phase flow is incompressible and the two-phase interface has a constant surface tension coefficient $\sigma$. The channel is considered isothermal, and the heat transfer in the flow channel is also neglected. The channel and MEA surfaces are considered impermeable. The associated coordinate system is also defined and given in Figure 2.

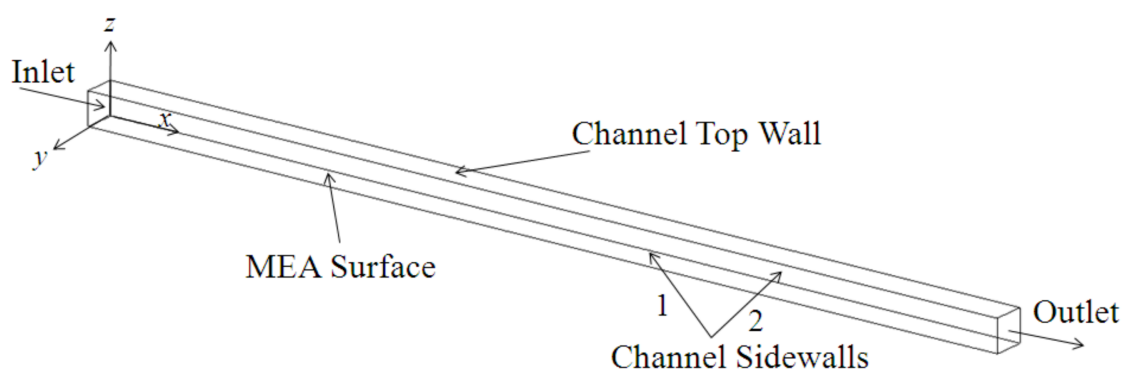

Figure 2. Schematic of the computational domain and associated coordinate system. 


\subsection{Governing Equations and the VOF Method}

The VOF method is used to capture the air-water interface for the two-phase flow in the flow channel, and the governing equations consist of only one set of conservation equations of the air-water mixture. The governing equations for the flow therefore can be written as:

Continuity equation

$$
\frac{\partial \rho}{\partial t}+\nabla \cdot(\rho \mathbf{V})=0
$$

Momentum equation

$$
\frac{\partial(\rho \mathbf{V})}{\partial t}+\nabla \cdot(\rho \mathbf{V} \mathbf{V})=-\nabla P+\mu \nabla \cdot\left(\nabla \mathbf{V}+\nabla \mathbf{V}^{\mathrm{T}}\right)+\rho \mathbf{g}+\mathbf{F}_{\mathbf{s}}
$$

in which $P$ is the static pressure, $\mu$ is the viscosity, $\mathbf{g}$ is the gravity, and $\mathbf{F}_{\mathbf{s}}$ is a source term, which represents an external force caused by the surface tension effect at the interface between the air-water two phases. With the VOF interface tracking method, this external force can be expressed as [30,31]:

$$
\mathbf{F}_{\mathbf{s}}=\sigma \frac{\rho \kappa \nabla f_{1}}{0.5\left(\rho_{1}+\rho_{2}\right)}
$$

in which $\sigma$ is the surface tension coefficient at the air-water interface, and the parameter $f_{1}$ represents the volume fraction of the fluid 1, i.e., liquid water. The subscript " 1 " here denotes the properties of the water phase and the subscript " 2 " for the properties of the air phase. Only two phases are involved, hence the volume fraction of the fluid 2, the air, can be determined by the following relationship:

$$
f_{1}+f_{2}=1
$$

All the properties appearing in the governing equations of the mixture level are computed in a volume fraction, weighted-average manner based on the individual phases; for example, the density and viscosity are obtained as:

$$
\rho=\rho_{1} f_{1}+\rho_{2} f_{2} \text { and } \mu=\mu_{1} f_{1}+\mu_{2} f_{2}
$$

The parameter $\kappa$ appearing in Equation (3) is the surface curvature at the two-phase interface, which can be expressed as:

$$
\kappa=\nabla \cdot \hat{\mathbf{n}}=\nabla \cdot \frac{\mathbf{n}}{|\mathbf{n}|}
$$

in which $\hat{\mathbf{n}}$ is the surface unit normal, and $\mathbf{n}$ is the surface normal represented by the phase volume fraction gradient:

$$
\mathbf{n}=\nabla f_{1}
$$

The wall adhesion influences the surface unit normal in the boundary cells, and hence the surface unit normal is adjusted in terms of the contact angle:

$$
\hat{\mathbf{n}}=\hat{\mathbf{n}}_{\mathbf{w}} \cos (\theta)+\hat{\mathbf{t}}_{\mathbf{w}} \sin (\theta)
$$

in which $\hat{\mathbf{n}}_{\mathbf{w}}$ and $\hat{\mathbf{t}}_{\mathbf{w}}$ are the unit vectors normal and tangential to the wall, respectively, and $\theta$ is the static contact angle of the liquid water at the wall [30]. Then, the surface curvature $\kappa$ of the interface in the boundary cells at the wall becomes:

$$
\kappa=\nabla \cdot \hat{\mathbf{n}}=\nabla \cdot\left[\hat{\mathbf{n}}_{\mathbf{w}} \cos (\theta)+\hat{\mathbf{t}}_{\mathbf{w}} \sin (\theta)\right]
$$


In order to obtain the phase volume fraction in the cells, a continuity-like equation is solved throughout the computational domain in the VOF method:

$$
\frac{\partial f_{1}}{\partial t}+\nabla \cdot\left(f_{1} \mathbf{V}\right)=0
$$

After the phase volume fraction is obtained, the air-water interface in the cells is constructed using the piecewise-linear approach according to the value of the phase volume fraction [30].

\subsection{Boundary and Initial Conditions}

At the channel inlet, a uniform velocity is applied, with the flow direction normal to the channel inlet. At the channel outlet, the flow is considered fully developed and the pressure is considered constant with the value of $P_{\text {out }}$. At the MEA and the channel surfaces, a no-slip wall boundary condition is used that applies a zero fluid velocity at the surfaces, except that a specified shear wall boundary condition is applied for the water spread on the surfaces that sets the wall shear stress on the water according to the sliding angle. The specified shear wall boundary condition is applied by using a user defined function. The boundary conditions for the simulation can be expressed mathematically as:

At the inlet:

$$
v_{x}=v_{x, 0}, v_{y}=v_{z}=0
$$

At the outlet:

$$
\frac{\partial v_{x}}{\partial x}=0, v_{y}=v_{z}=0, P=P_{\text {out }}
$$

At the MEA and channel surfaces:

$$
v_{x}=v_{y}=v_{z}=0
$$

Except for the MEA and channel surfaces, which are in contact with the liquid water:

$$
\mu \frac{d v_{x}}{d z}=\tau_{x}=\frac{F_{w}}{A}=\frac{m g \sin (\alpha)}{A}, \mu \frac{d v_{y}}{d z}=\tau_{y}=0, v_{z}=0
$$

in which $v$ is the velocity component, $\tau$ is the wall shear stress component, $F_{\mathrm{w}}$ is the wall adhesion force shown in Figure 1, and $A$ is the water spreading area on solid surface, which can be retrieved from the numerical result and used for the calculation of wall shear stress.

Initially (or at the time $t=0$ ), a water droplet is introduced on the MEA surface, with the diameter of $D_{\text {droplet }}, 1 \mathrm{~mm}$ downstream of the channel inlet. The initialized velocity and the gauge pressure are both set as zero for the computational domain. At $t>0$, a uniform air velocity of $v_{\mathrm{x}, 0}$ is applied at the channel inlet. The absolute pressure is maintained as $P_{\text {out }}$ at the channel outlet. The gravity force is considered along the negative $\mathrm{z}$-direction, and the contact angle and sliding angle are set at the MEA and channel surfaces, representing the surface wettability.

\subsection{Numerical Technique and Grid}

The ANSYS FLUENT CFD platform is employed to run the simulation. The control volume-based technique is used to discretize the governing equations. The pressure-based solver is used for the unsteady flow solution, with the Green-Gauss cell-based gradient evolution and the first order implicit temporal discretization. The pressure implicit with split operator (PISO) scheme is selected for the pressure-velocity coupling, and the second-order upwind scheme is selected for the momentum equation. An explicit scheme-based VOF method is used to track the interface of the two-phase flow, and the interface between the two phases is constructed using the piecewise linear Geo-Reconstruct scheme based on the fluid volume fraction in the cells. The time step is selected as $10^{-6} \mathrm{~s}$. The hexahedral grids are used for meshing, and the grid number is selected as $30 \times 30 \times 30$ (grid number per millimeter for $\mathrm{x} \times \mathrm{y} \times \mathrm{z}$ ), with 810,000 total grids discretizing the whole computational 
domain. The grid setup in this study is similar to the previous study and is considered reasonable according to [23-25], in which the water distribution and the pressure drop were checked, and the difference was very small owing to the substitution of the grid number for the grid independence study.

\section{Results and Discussion}

In all the cases investigated, the air inlet velocity $v_{x, 0}$ is selected as $6 \mathrm{~m} \cdot \mathrm{s}^{-1}$, with the Reynolds number of about 400. The water droplet introduced on the MEA surface has a diameter of $0.6 \mathrm{~mm}$. The air-water two-phase interface has a constant surface tension coefficient $\sigma$ of $0.073 \mathrm{~N} \cdot \mathrm{m}^{-1}$. The MEA surface is hydrophobic with a contact angle $\theta_{\mathrm{MEA}}$ of $140^{\circ}$ and a sliding angle $\alpha_{\mathrm{MEA}}$ of $30^{\circ}$, representing the typical values of the MEA surface [29]. The absolute channel outlet pressure $P_{\text {out }}$ is maintained as $1 \mathrm{~atm}$. The effect of water droplet location and channel surface wettability on the water dynamics in the flow channel are investigated in the following two sections, respectively. All the cases investigated are given in Table 1.

Table 1. Computational cases and parameter values considered in the present study. $D$ is the distance from the water spreading center on the MEA surface to the MEA central line, representing the initial water droplet location; $\theta_{\text {channel }}$ is the channel surface contact angle; and $\alpha_{\text {channel }}$ is the channel surface sliding angle, representing the channel surface wettability.

\begin{tabular}{cccc}
\hline Cases & $\boldsymbol{D} \mathbf{( \mathbf { m m } )}$ & $\boldsymbol{\theta}_{\text {channel }}\left({ }^{\circ}\right)$ & $\boldsymbol{\alpha}_{\text {channel }}\left(^{\circ}\right)$ \\
\hline Conventional case & 0 & 70 & 90 \\
Case 1 & 0.05 & 70 & 90 \\
Case 2 & 0.1 & 70 & 90 \\
Case 3 & 0.15 & 70 & 90 \\
Case 4 & 0.15 & 45 & 90 \\
Case 5 & 0.15 & 110 & 53 \\
Case 6 & 0.15 & 140 & 30 \\
\hline
\end{tabular}

\subsection{Effect of the Water Droplet Location}

Figure 3 shows an illustration of the locations where water droplet is introduced along the channel width direction (y direction) on the MEA surface. The water droplet location is described by a parameter $D$, the distance from the water spreading center to the MEA central line on the MEA surface. The water droplet location $D$ can vary from $0-0.2 \mathrm{~mm}$, since the water droplet diameter is $0.6 \mathrm{~mm}$ and the water droplet is not in contact with the channel surface initially. Therefore, the water droplet locations considered include $D$ equal to $0,0.05,0.1$, and $0.15 \mathrm{~mm}$, respectively, corresponding to the conventional case and Case 1-3 given in Table 1. For the cases investigated in this section, the spreading center of the water droplet introduced is located only at one half of the MEA surface separated by the MEA central line, which is adjacent to the channel sidewall 1 , since the flow channel is symmetric with regard to the channel middle plane. All the channel surfaces are hydrophilic, with a contact angle $\theta_{\text {channel }}$ of $70^{\circ}$ and a sliding angle $\alpha_{\text {channel }}$ of $90^{\circ}$. Figure 4 shows a comparison of water transport and dynamics in the flow channel between the conventional case with $D=0$ and Case 3 with $D=0.15 \mathrm{~mm}$. For the conventional case as shown in Figure 4a, it is seen that the water droplet is only transported along the MEA surface, in contact with no channel surface throughout its transport in the flow channel. The water spreading center for this case is found always located on the MEA central line, as shown in Figure 5a, due to the symmetry of water transport in the flow channel. However, for Case 3 with the largest initial water distance $D$ to the MEA center as shown in Figure $4 \mathrm{~b}$, it is found that the water droplet is also transported along the channel width direction. Although it is initially introduced more closely to the channel sidewall 1 , the water droplet is transported to the other side, approaching the channel sidewall 2, which is more clearly shown in Figure 5d. The water droplet reaches the hydrophilic channel sidewall 2 at about $4.7 \mathrm{~ms}$. Then, due to the relatively high capillary wicking effect of the channel surface, the water is seen to be dragged to the channel sidewall 2 , stretched, and spread out on the 
channel sidewall 2 while shrinking its contact area on the MEA surface. At about $6.5 \mathrm{~ms}$, the water droplet is seen to detach completely from the MEA surface. In the meantime, the water continues spreading on the channel sidewall 2 until it reaches the channel top surface at about $7.3 \mathrm{~ms}$. Then, due to the strong capillary wicking effect at the channel corner, the water droplet is contracted to the channel corner formed by the channel sidewall 2 and the channel top surface, and transported downstream along the channel corner from then on. Water removal from the MEA surface for Case 3 is desirable, since it quickly and effectively clears the passage for the reactant gas transport to the reaction sites in the MEA. It reveals that the gas flow channel itself has MEA surface liquid water cleaning ability for the water emerging far away from the MEA center, as in Case 3.

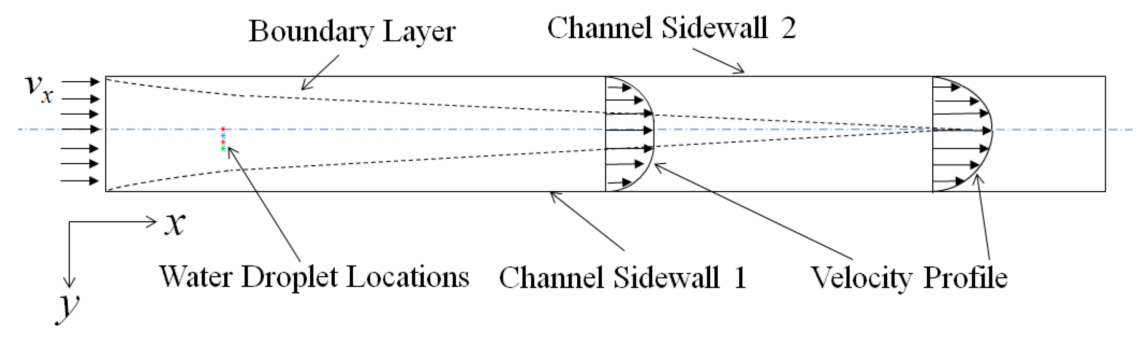

Figure 3. Illustrations of the initial water droplet locations, gas boundary layer, and velocity profiles in the middle $\mathrm{z}$ plane of the gas flow channel. The water droplet location is represented by the water spreading center on the MEA surface.

The water spreading on the MEA surface for the cases investigated is presented in Figure 5. It clearly shows that the water droplet oscillates along the channel width direction in the flow channel for both Case 1 with $D=0.05 \mathrm{~mm}$ and Case 2 with $D=0.1 \mathrm{~mm}$, with the oscillation amplitude increasing with $D$. It is also noticed that the water oscillation amplitude decreases with time, which is attributed to the dissipation of the oscillation energy due to the frictional loss. The water oscillation is damped quickly, and the water spreading center converges to the MEA center for both Case 1 and Case 2. Since the water oscillation amplitude is the largest for the first period of the oscillation, the water droplet will never reach the hydrophilic channel surface if it cannot touch the channel surface in the first period. The water spreading area on the MEA surface is also seen to experience a wave-like fluctuation, accompanied by its shape fluctuation. It is also found that the water droplet has almost the same velocity along the channel length direction ( $x$ direction) for the conventional case, Case 1 and Case 2, reflected by the water droplet location with time along the channel length direction, indicating that the water droplet primary velocity is hardly affected by the initial water location along the channel width direction.

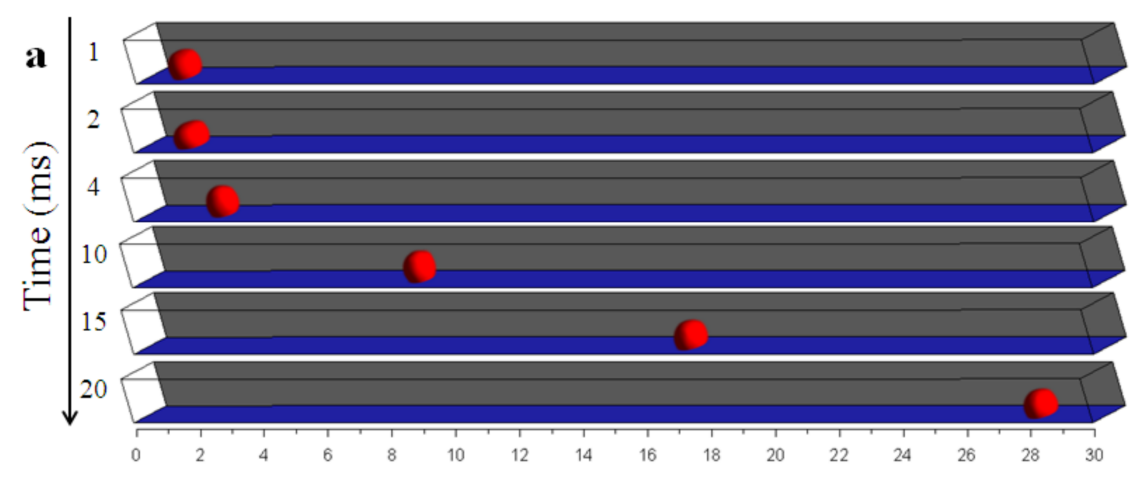

Figure 4. Cont. 


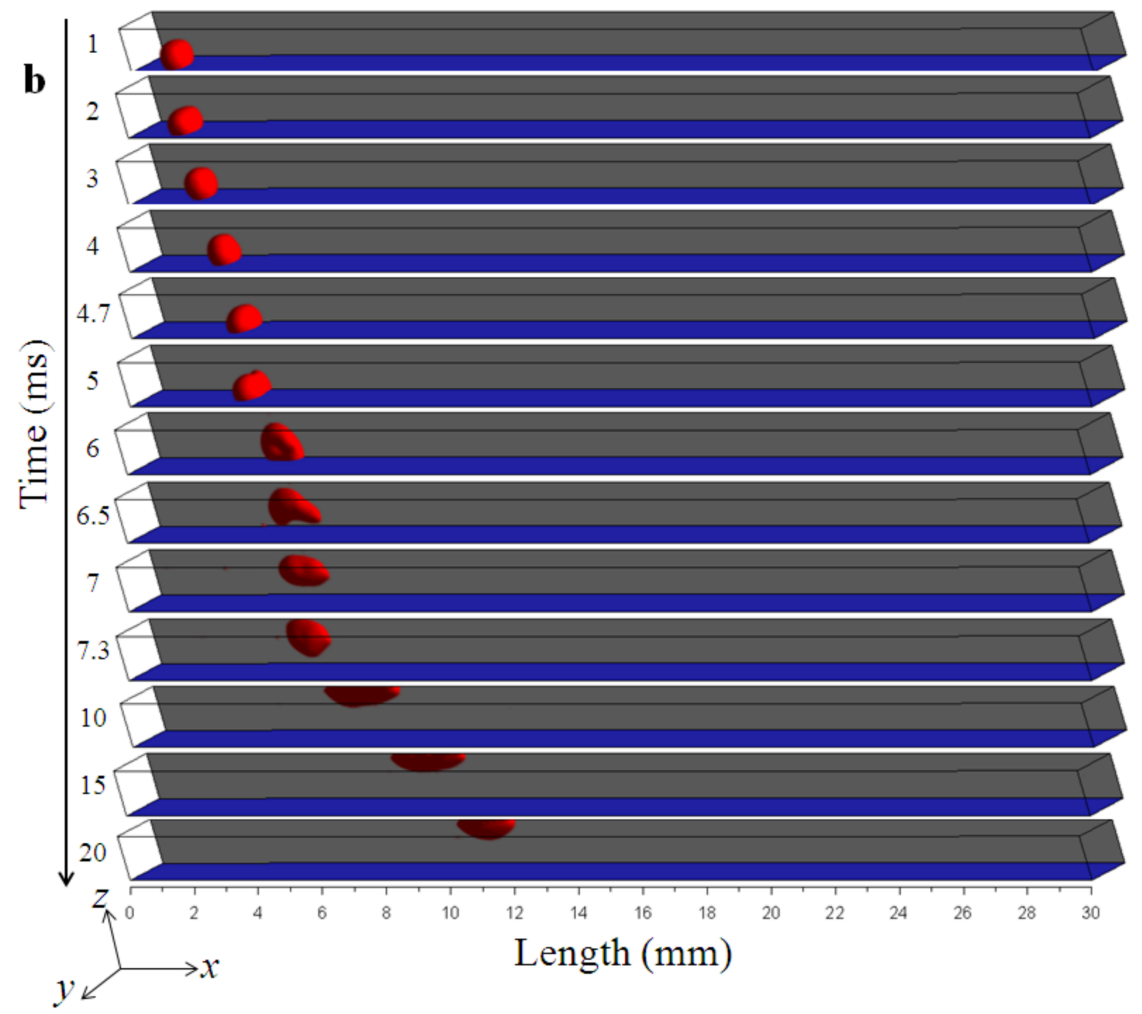

Figure 4. Water transport and dynamics in the flow channel with the initial water location (a) $D=0$, corresponding to the conventional case, and (b) $D=0.15 \mathrm{~mm}$, corresponding to Case 3 given in Table 1 . $D$ is the distance from the water spreading center to the MEA central line on the MEA surface.

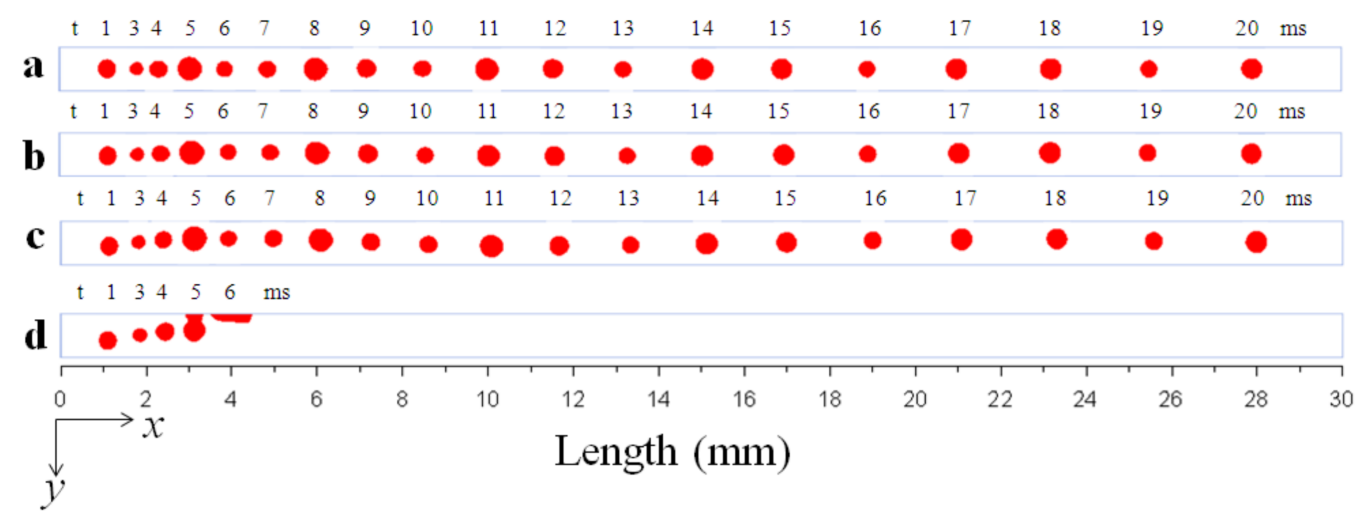

Figure 5. Water spreading on the MEA surface for various water initial locations in the flow channel: (a) $D=0$, the conventional case; (b) $D=0.05 \mathrm{~mm}$, Case 1 ; (c) $D=0.1 \mathrm{~mm}$, Case 2; (d) $D=0.15 \mathrm{~mm}$, Case 3. $D$ is the distance from the water spreading center to the MEA central line on the MEA surface.

As is known, the flow is laminar in the gas flow channel. The laminar boundary layer thickness grows as the distance to the channel inlet is increased up to a constant value (half channel width or height) when the flow is fully developed, as shown in Figure 3. Due to the existence of the boundary layer, the flow velocity magnitude is not uniform at the channel cross section (yz plane); the flow velocity is larger when closer to the channel center and is smaller when otherwise closer to the channel surface. When the water droplet is introduced at the center of the MEA surface for the conventional case, the air flow velocity is seen to be symmetric about the water droplet shown in Figure 6a, leading to the symmetric air shear force at both the sides of the water droplet (y direction), so the water droplet bears a zero net driving force along the channel width direction and is only transported along the 
central line of the MEA surface. In contrast, when the water droplet is introduced (deviating from the center of the MEA surface for Case 1-3), the air velocity is seen to be asymmetric with regard to the water droplet shown in Figure $6 \mathrm{~b}-\mathrm{d}$, leading to a larger air shear force acting on the water droplet at the side closer to the central line of the MEA surface, such that the water droplet bears a net driving force that is always pointing to the center of the MEA surface, and the water droplet can be transported along the channel width direction. The pressure is asymmetric about the water droplet shown in Figure $6 \mathrm{~b}-\mathrm{d}$, which also contributes to the net driving force pointing to the center of the MEA surface. It is also observed that the flow velocity and pressure difference, namely the net driving force along the channel width direction, is larger as the water droplet introduced deviates more from the center of the MEA surface (with a larger $D$ ), so the water oscillation amplitude increases as $D$ is increased. Therefore, only the water droplet for Case 3 with the largest $D$ is driven to the hydrophilic channel surface by the largest net air driving force along the channel width direction and removed from the MEA surface afterward, whereas the water droplet for Case 1 and Case 2 with smaller $D$ ends up with the oscillation on the MEA surface in the flow channel. Similarly, the wave-like fluctuation of the water droplet shape (or height along $\mathrm{z}$ direction) can also be explained by the asymmetric air velocity or air shear force acting on the water droplet. Under such a kind of air shear force, water droplet can even roll on the hydrophobic surface, which has been observed in the experimental study [32].

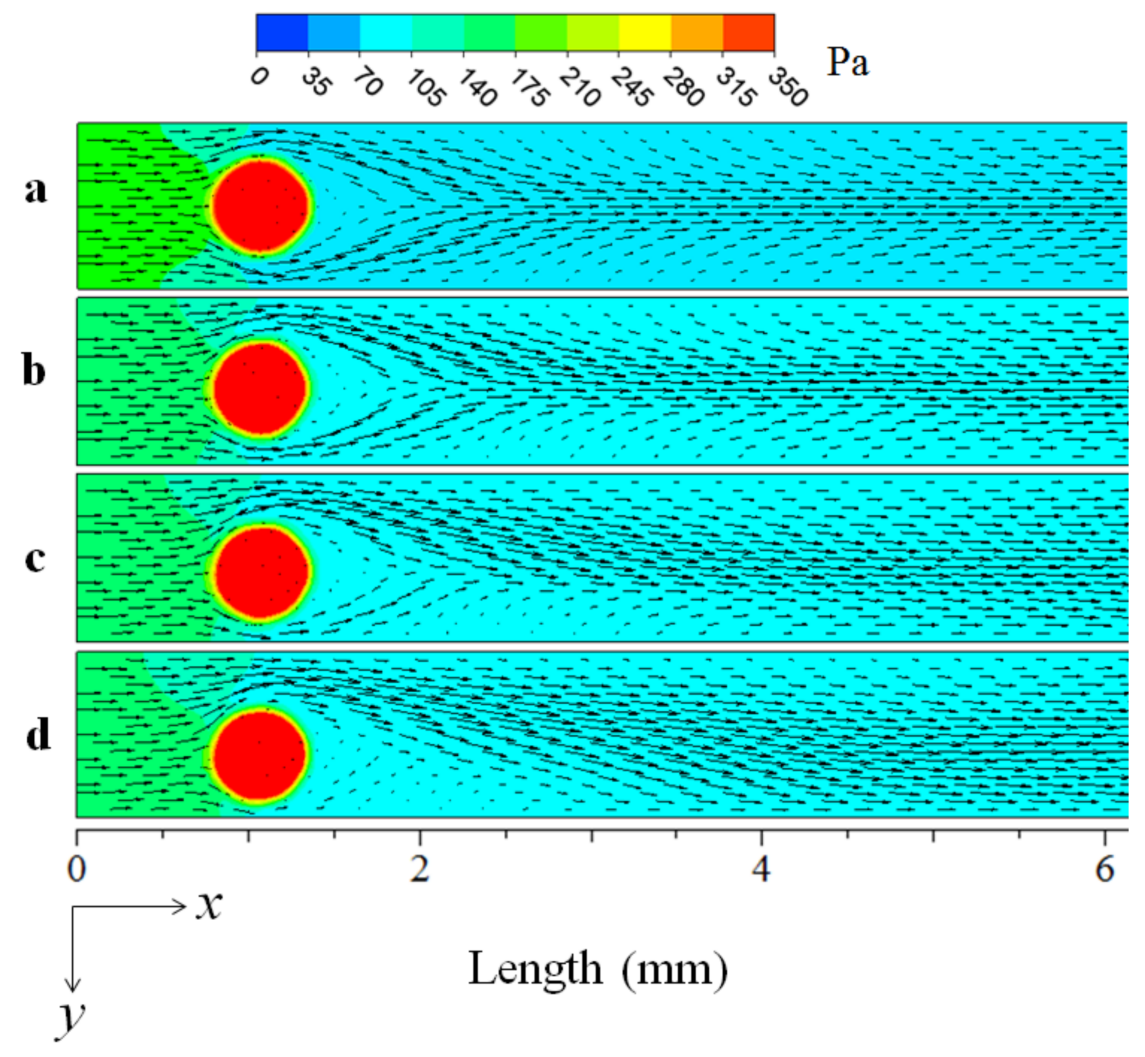

Figure 6. Flow field (velocity plot) and pressure in the cross sectional plane of the flow channel having various initial water locations at the time instant of $1 \mathrm{~ms}$, with the plane distance of $0.3 \mathrm{~mm}$ to the MEA surface: (a) $D=0$, the conventional case; (b) $D=0.05 \mathrm{~mm}$, Case 1 ; (c) $D=0.1 \mathrm{~mm}$, Case 2; (d) $D=0.15 \mathrm{~mm}$, Case 3. $D$ is the distance from the water spreading center to the MEA central line on the MEA surface.

In order to better clarify the advantage of the improved model, simulations are also carried out with the no-slip boundary condition for comparisons. It is seen from Figure 7 that the wall shear stress for water droplet at the wall is about $2.5 \mathrm{~Pa}$ with the slip boundary condition, which is consistent with the value calculated from Equation (14). However, the wall shear stress for water droplet is 
quite large with the no-slip boundary condition. It is pointed out that the maximum shear stress with the no-slip boundary condition is about $40 \mathrm{~Pa}$, with the values exceeding the maximum scale in the legend compressed into the red color in the region covered by the water droplet, as shown in Figure 7a. The no-slip boundary condition forces the velocity at the wall to reduce to zero and exerts an excessive, large resistant force on the water droplet. As a result, the water flow velocity is smaller than that with the slip boundary condition, as shown in Figure $7 b, c$, compared with the corresponding results given in Figure 5. The water droplet would not even reach the channel sidewall and be removed for $D$ of $0.15 \mathrm{~mm}$, due to the large resistant force, as shown in Figure 7c. The large resistant force from the wall stretches the water spreading on the MEA surface and also restricts the water shape oscillation, with the water spreading area variation smaller than for the slip boundary condition. It is stressed that the no-slip boundary condition greatly magnifies the wall shear stress on water droplet and may not apply well for sliding flow on hydrophobic surfaces in PEMFC [28].

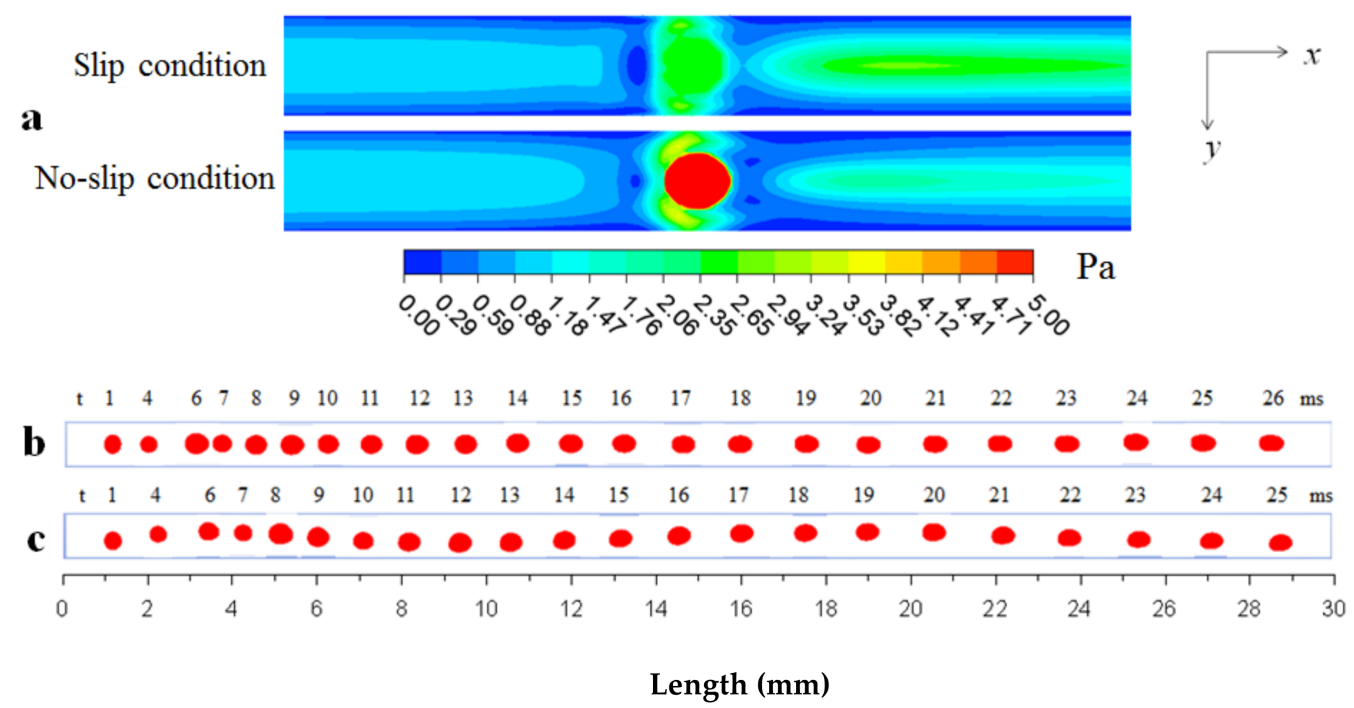

Figure 7. Comparisons of water transport in the flow channel: (a) wall shear stress at the MEA surface between the simulations with slip (the conventional case in Table 1) and no-slip boundary conditions at $14 \mathrm{~ms}$; (b) water spreading on the MEA surface for $D=0$ with the no-slip condition; (c) water spreading on the MEA surface for $D=0.15 \mathrm{~mm}$ with the no-slip condition. Other conditions are the same, with the corresponding slip cases given in Table 1 .

The pressure drop for the flow in the channel is given in Figure 8. It is found that the pressure drop is almost the same for the conventional case, Case 1, and Case 2, in which the water droplet is only transported on the MEA surface, whereas the pressure drop is decreased significantly after the water droplet reaches the hydrophilic channel surface for Case 3. It reveals that the pressure drop is hardly influenced by the initial water location if the water is transported only on the MEA surface. The pressure drop is smaller for Case 3, because water is thinned out on the hydrophilic channel surface, increasing the available cross section for the air to pass through and decreasing the flow blockage in the flow channel. It also shows that the pressure drop has fluctuations, which are associated with the water spreading area fluctuations presented in Figure 5. The pressure drop analysis reveals that the pressure drop is a good indicator for water transport and distribution in the flow channel. 


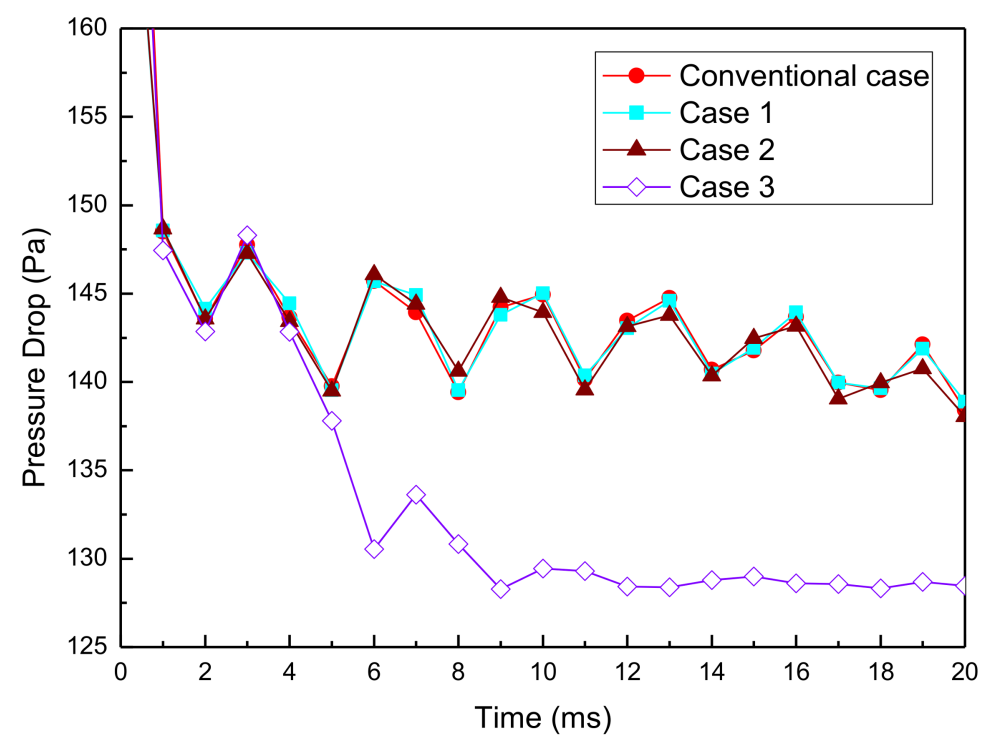

Figure 8. Pressure drop for the flow in the flow channel with various water droplet locations for the cases given in Table 1 .

\subsection{Effect of the Channel Surface Wettability}

The channel surface wettability significantly influences water removal from the MEA surface [22]. In order to reveal its effect on water removal, the channel surface wettability is investigated considering the sliding angle impact. The channel surface wettability considered includes the contact angle $\theta_{\text {channel }}$ equal to $70^{\circ}, 45^{\circ}, 110^{\circ}$, and $140^{\circ}$, respectively, corresponding to Case 3-6 shown in Table 1 . The associated sliding angle $\alpha_{\text {channel }}$ for the channel surface is also considered and obtained from a correlation between the contact angle and the sliding angle widely adopted in the previous studies $[33,34]$, as shown below:

$$
k=\left\{\frac{9 V^{2}\left[2-3 \cos (\theta)+\cos ^{3}(\theta)\right]}{\pi^{2}}\right\}^{1 / 3} \frac{\rho g \sin (\alpha)}{6 \sin (\theta)}
$$

in which $V$ is the volume of the water droplet and $k$ is a constant related to the interaction energy between solid and liquid. However, this correlation is only applicable to the hydrophobic surface, and for the hydrophilic channel surface where the contact angle is smaller than $90^{\circ}$, the sliding angle is assumed to be $90^{\circ}$, corresponding to the largest value in the present study. As for the hydrophobic channel surface, the constant $k$ in this correlation is determined by using the contact angle and sliding angle values of the MEA surface obtained from a previous experimental study [29], and then the sliding angle for the channel surface is calculated by this correlation based on the given channel surface contact angle. The associated sliding angles for the cases investigated are also given in Table 1 . For the cases investigated in this section, the water droplet location $D$ is fixed as $0.15 \mathrm{~mm}$. The channel surface wettability only takes effect after the water droplet reaches the channel surface, which could have considerable impact on the subsequent water corner flow in the flow channel.

Figure 9 presents the simulated results of water transport and dynamics in the flow channel with the channel surface contact angle of $45^{\circ}$, corresponding to Case 4 given in Table 1 . It is found that for this case with more hydrophilic channel surface, the water is spread more widely on the channel sidewall 2, and it reaches the channel top surface first at about $6.5 \mathrm{~ms}$, which is much faster than for Case 3. The water is seen to be detached completely from the MEA surface at about $6.6 \mathrm{~ms}$, which is slightly slower than for Case 3. Then, similar to Case 3, the water is contracted to the channel corner formed by the channel sidewall 2 and the channel top surface, due to the strong capillary force there. It is also noticed that the water is thinner and the water flow velocity is smaller along the channel corner compared to Case 3, which is attributed to the more hydrophilic channel surface for this case. 


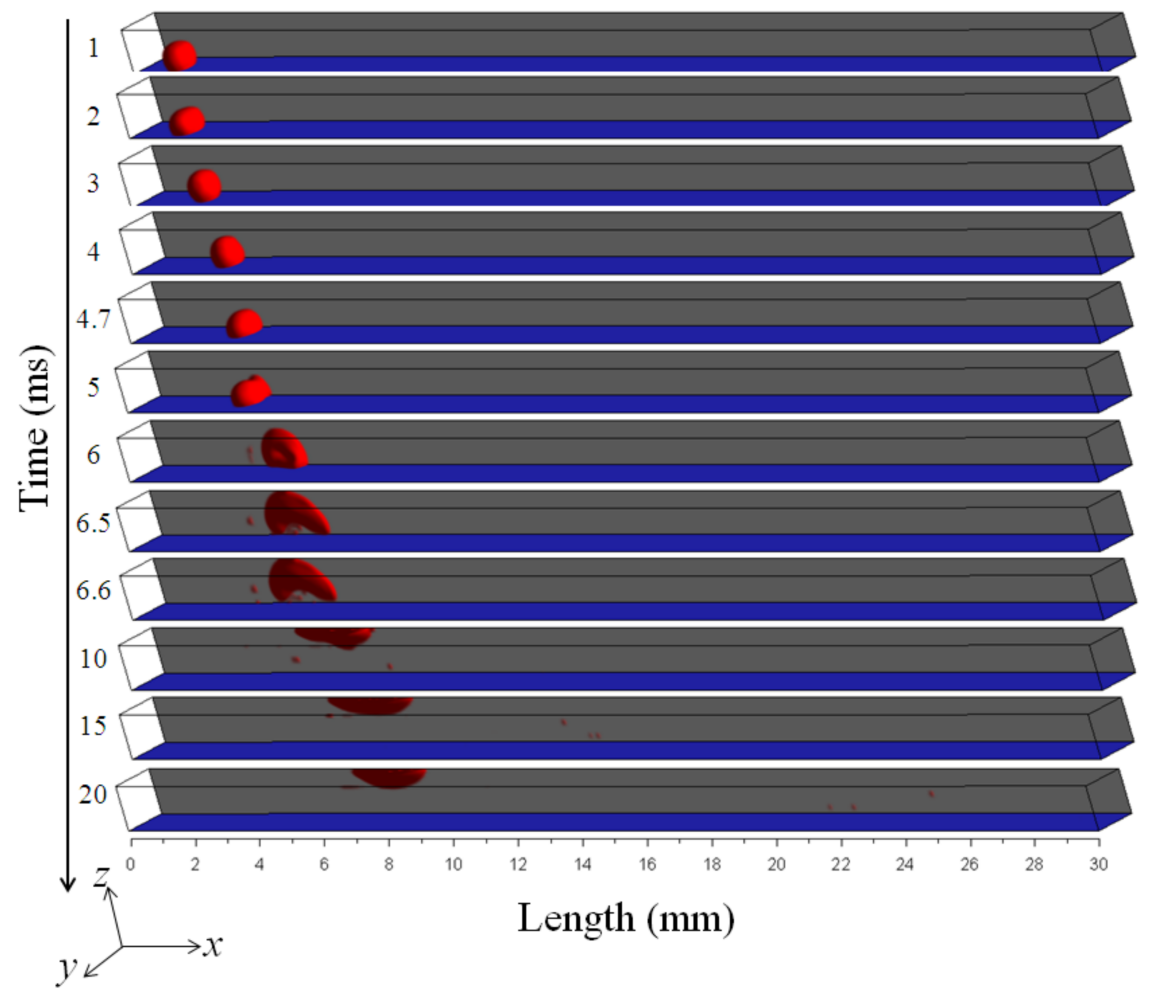

Figure 9. Water transport and dynamics in the flow channel with the channel surface contact angle of $45^{\circ}$, corresponding to Case 4 given in Table 1 .

On the other hand, shown in Figure 10 is the water transport and dynamics in the flow channel with the channel surface contact angle of $110^{\circ}$, corresponding to Case 5 given in Table 1 . It is found that the water droplet cannot be removed from the MEA surface by the weak capillary force of the hydrophobic channel surface for this case. The water droplet is seen to be contracted to the channel corner formed by the MEA surface and the channel sidewall 2, rather than that formed by the channel sidewall 2 and the channel top surface. It is also noticed that the water spreading area on the channel sidewall 2 is larger than that on the MEA surface, since the channel sidewall 2 is less hydrophobic than the MEA surface. Figure 11 shows the water transport and dynamics in the channel for Case 6 with the channel surface contact angle of $140^{\circ}$, which is identical to the contact angle of the MEA surface. It is found that for this case, the water spreading area on the channel sidewall 2 is almost the same as that on the MEA surface, since the channel surface and the MEA surface are equally hydrophobic this time. As is seen, the water for Case 6 is transported faster than that for Case 5 shown in Figure 10, due to the more hydrophobic channel surface.

The pressure drop for the flow in the channel is given in Figure 12. It is found that the pressure drop is the same for the cases investigated before the water droplet reaches the channel surface. The pressure drop for all the cases is decreased considerably after the water droplet reaches the channel surface. The pressure drop is seen to be decreased as the contact angle of the channel surface is decreased. This is because the water is thinner on a more hydrophilic surface, reducing the blockage for the flow in the channel. It is also found that the pressure drop fluctuation, reflecting the water shape fluctuation, is decreased with the channel surface contact angle. 


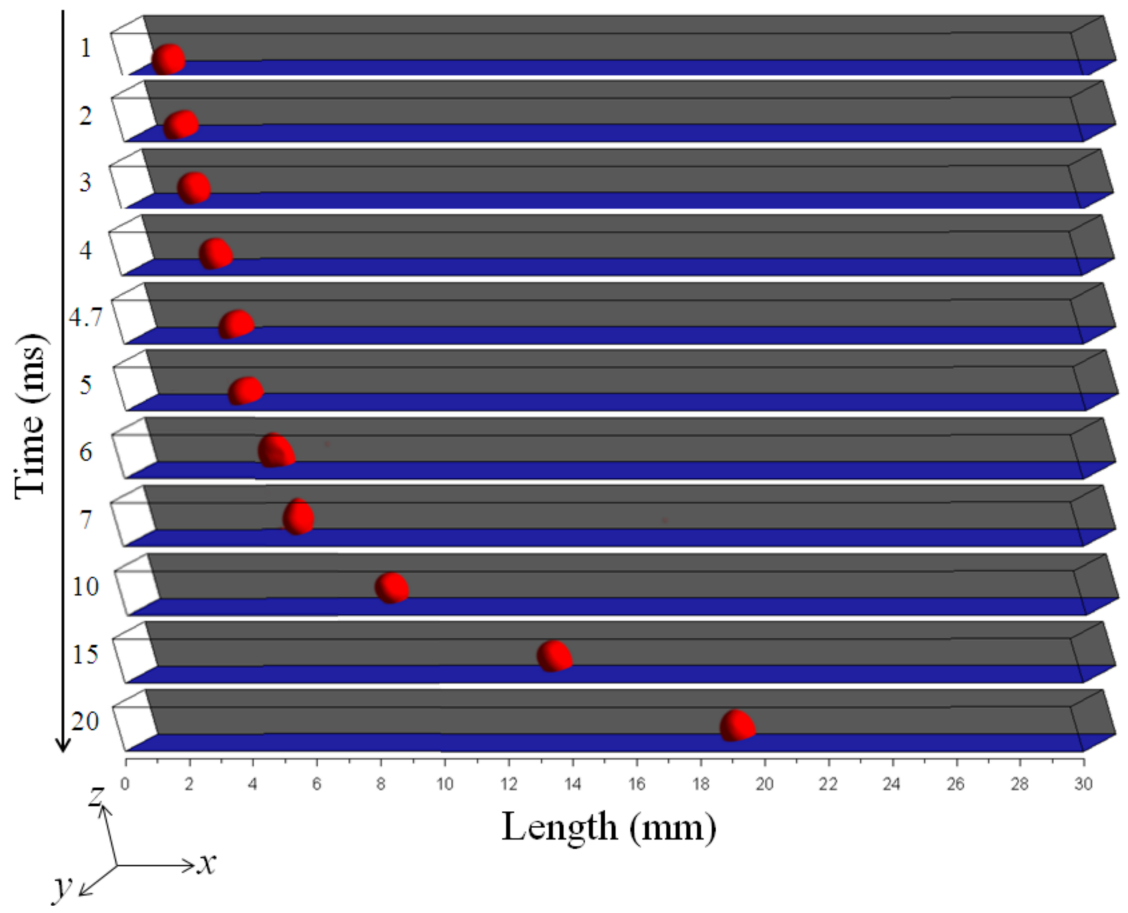

Figure 10. Water transport and dynamics in the flow channel with the channel surface contact angle of $110^{\circ}$, corresponding to Case 5 given in Table 1.

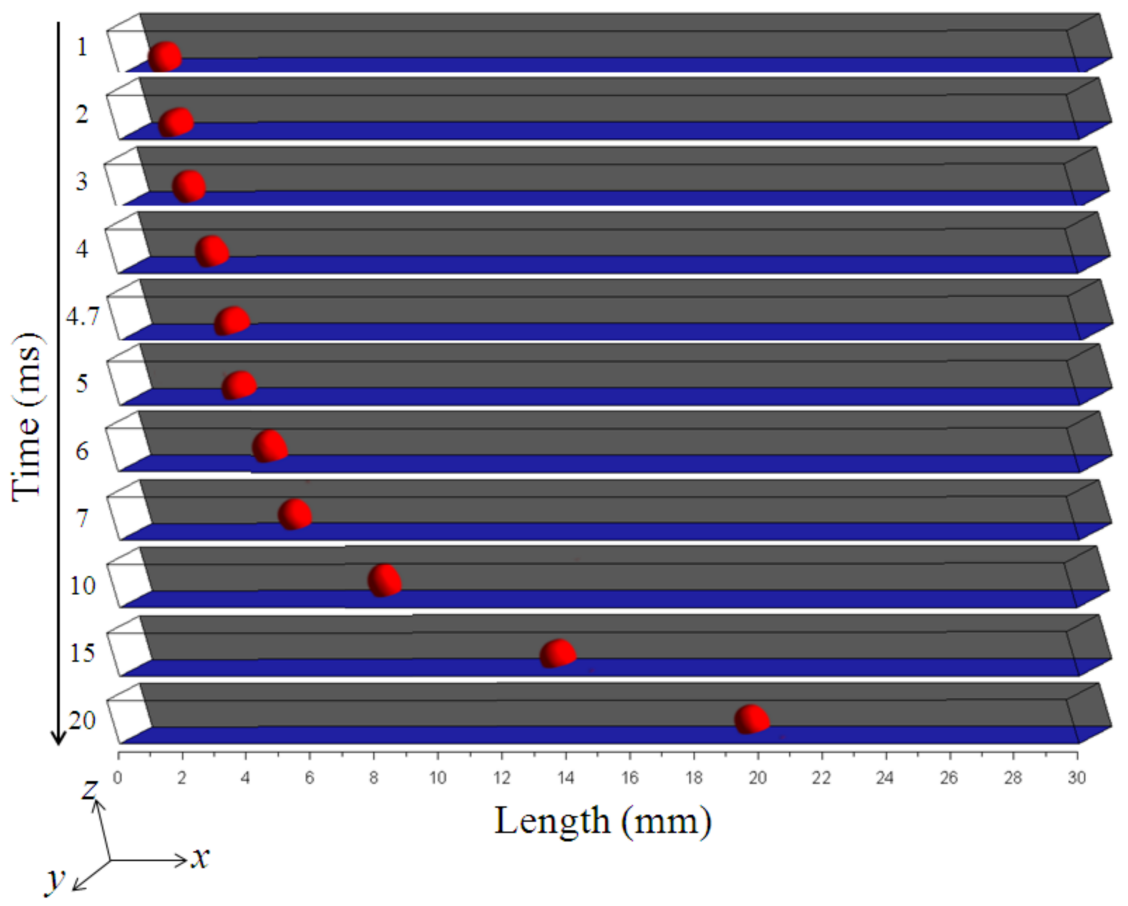

Figure 11. Water transport and dynamics in the flow channel with the channel surface contact angle of $140^{\circ}$, corresponding to Case 6 given in Table 1. 


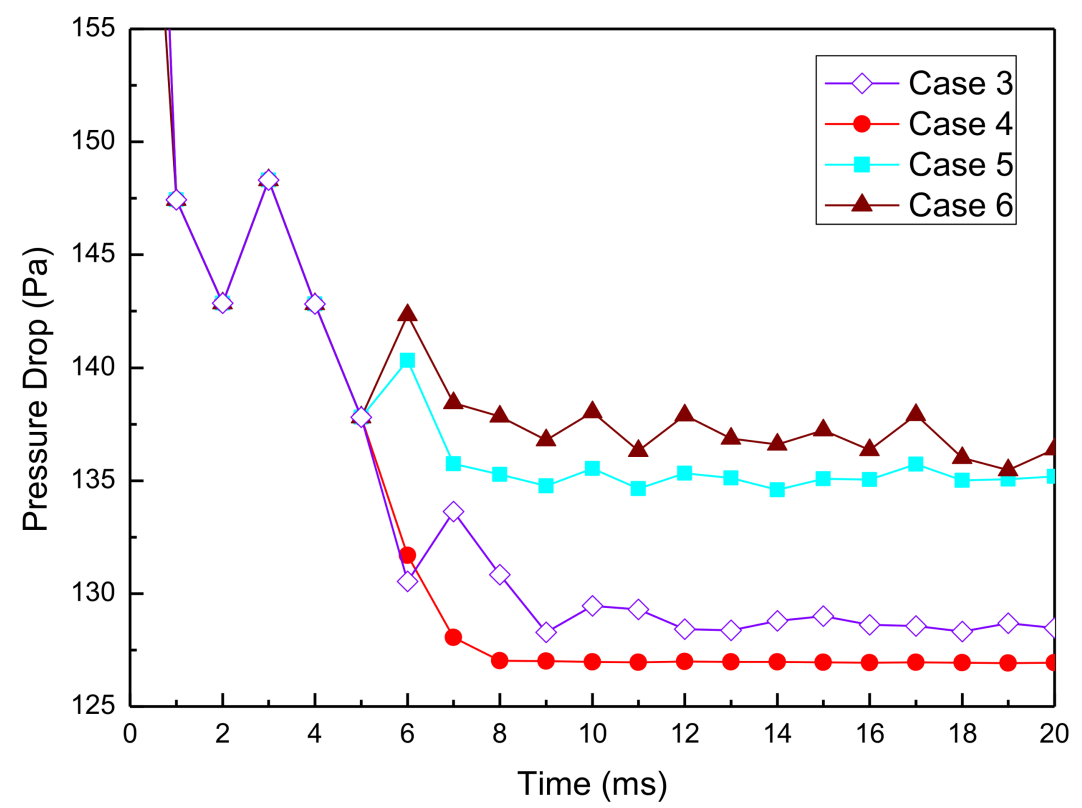

Figure 12. Pressure drop for the flow in the flow channel with various channel surface wettability for the cases given in Table 1.

Through comparisons of water transport and removal in the flow channel with various channel surface wettabilities, it is clear that the channel surface wettability has significant influence on the water corner flow in the flow channel. In order to remove the water from the MEA surface accumulated in the channel corner between the MEA surface and the channel surface, the channel surface contact angle must be small enough, resulting in a sufficiently strong capillary force acting on the water, to overcome the wall adhesion force from the MEA surface. On the other hand, excessive small channel surface contact angle does not accelerate the water removal from the MEA surface but leads to slow water corner flow in the flow channel, impeding the water removal process in the flow channel. Indeed, if a large amount of liquid water is produced in PEMFC, water annular and water slug flow can be easily formed in the flow channel with excessive hydrophilic channel surfaces, blocking the reactant gas transport heavily [12]. Considering both the effective water removal from the MEA surface and water removal from the flow channel, as well as an acceptable pressure drop in the flow channel, the channel surface wettability must be carefully selected based on the design and operating conditions of PEMFC. In this study, the channel surface with the contact angle of $70^{\circ}$ seems to be the best among the cases investigated.

\section{Conclusions}

In this study, water transport and removal in a proton exchange membrane fuel cell (PEMFC) gas flow channel with various water droplet locations and channel surface wettability has been investigated numerically by using the volume-of-fluid (VOF) method incorporating the water sliding flow property, in which both the contact angle and the sliding angle are considered for the surface wettability. It is found that the water droplet oscillates along the channel width direction when the water droplet does not emerge at the center of the membrane-electrode assembly (MEA) surface, and that the water droplet reaches the channel surface, forming the water corner flow in the flow channel, when the water emerging location is far away enough from the MEA center. The water corner flow is influenced significantly by the channel surface wettability. The water accumulated at the channel corner formed by the MEA surface and the channel surface is removed by the channel surface when the channel surface is hydrophilic enough. The channel surface that has a contact angle of $70^{\circ}$ is the 
most desirable in the present study for effective water removal and the small pressure drop for the flow in the flow channel.

Acknowledgments: This work is financially supported by the National Natural Science Foundation of China (Grant No. 51706153).

Author Contributions: All authors have contributed to this research work. Yanzhou Qin conceived research ideas and contributed to the coding and manuscript; Xuefeng Wang and Rouxian Chen contributed to the model setup; Xiang Shangguan contributed to the data processing.

Conflicts of Interest: The authors declare no conflict of interest.

\section{Nomenclature}

\begin{tabular}{|c|c|}
\hline$A$ & water spreading area $\left(\mathrm{m}^{2}\right)$ \\
\hline$D$ & water location $(\mathrm{m})$ \\
\hline$D_{\text {droplet }}$ & water droplet diameter (m) \\
\hline$f$ & volume fraction of the fluid \\
\hline $\mathbf{F}_{\mathrm{s}}$ & external force term $\left(\mathrm{N} \cdot \mathrm{m}^{-3}\right)$ \\
\hline$F_{\mathrm{w}}$ & wall adhesion force $(\mathrm{N})$ \\
\hline $\mathbf{g}, g$ & gravitational force $\left(\mathrm{m} \cdot \mathrm{s}^{-2}\right)$ \\
\hline$k$ & constant in a correlation \\
\hline $\mathbf{n}$ & surface normal \\
\hline$\hat{\mathbf{n}}$ & surface unit normal \\
\hline$P$ & pressure term $(\mathrm{Pa})$ \\
\hline$t$ & Time \\
\hline$v$ & velocity component $\left(\mathrm{m} \cdot \mathrm{s}^{-1}\right)$ \\
\hline$V$ & volume $\left(\mathrm{m}^{3}\right)$ \\
\hline $\mathbf{V}$ & velocity vector $\left(\mathrm{m} \cdot \mathrm{s}^{-1}\right)$ \\
\hline \multicolumn{2}{|l|}{ Greek } \\
\hline$\alpha$ & sliding angle $\left(^{\circ}\right)$ \\
\hline$\theta$ & contact angle $\left(^{\circ}\right)$ \\
\hline$\kappa$ & surface curvature \\
\hline$\mu$ & viscosity $\left(\mathrm{kg} \cdot \mathrm{m}^{-1} \cdot \mathrm{s}^{-1}\right)$ \\
\hline$\rho$ & density $\left(\mathrm{kg} \cdot \mathrm{m}^{-3}\right)$ \\
\hline$\sigma$ & surface tension coefficient $\left(\mathrm{N} \cdot \mathrm{m}^{-1}\right)$ \\
\hline$\tau$ & wall shear stress component $(\mathrm{Pa})$ \\
\hline \multicolumn{2}{|c|}{ Subscripts } \\
\hline 0 & Initial \\
\hline 1,2 & liquid water, air \\
\hline a & advancing \\
\hline channel & gas flow channel \\
\hline droplet & water droplet \\
\hline MEA & the membrane electrode assembly surface \\
\hline Out & outlet \\
\hline $\mathrm{r}$ & receding \\
\hline S & source term \\
\hline $\mathrm{T}$ & transpose \\
\hline $\mathrm{w}$ & wall \\
\hline $\mathrm{x}, \mathrm{y}, \mathrm{z}$ & axes \\
\hline
\end{tabular}

\section{References}

1. Jiao, K.; Li, X. Water transport in polymer electrolyte membrane fuel cells. Prog. Energy Combust. Sci. 2011, 37, 221-291. [CrossRef]

2. Ji, M.; Wei, Z. A review of water management in polymer electrolyte membrane fuel cells. Energies 2009, 2, 1057-1106. [CrossRef] 
3. Bazylak, A. Liquid water visualization in PEM fuel cells: A review. Int. J. Hydrogen Energy 2009, 34, 3845-3857. [CrossRef]

4. Le, A.D.; Zhou, B. Fundamental understanding of liquid water effects on the performance of a PEMFC with serpentine-parallel channels. Electrochim. Acta 2009, 54, 2137-2154. [CrossRef]

5. Kim, B.; Lee, Y.; Woo, A.; Kim, Y. Effects of cathode channel size and operating conditions on the performance of air-blowing PEMFCs. Appl. Energy 2013, 111, 441-448. [CrossRef]

6. Park, J.; Oh, H.; Lee, Y.I.; Min, K.; Lee, E.; Jyoung, J.Y. Effect of the pore size variation in the substrate of the gas diffusion layer on water management and fuel cell performance. Appl. Energy 2016, 171, 200-212. [CrossRef]

7. Zhang, J.; Li, H.; Shi, Z.; Zhang, J. Effects of hardware design and operation conditions on PEM fuel cell water flooding. Int. J. Green Energy 2010, 7, 461-474. [CrossRef]

8. Lee, C.Y.; Fan, W.Y.; Chang, C.P. Micro humidity sensor for monitoring water flooding in a proton exchange membrane fuel cell. Int. J. Green Energy 2012, 9, 389-397. [CrossRef]

9. Park, Y.H.; Caton, J.A. Monitoring an electrode flooding through the back pressure in a proton exchange membrane (PEM) fuel cell. Int. J. Green Energy 2008, 5, 347-359. [CrossRef]

10. Li, Y.; Pei, P.; Wu, Z.; Xu, H.; Chen, D.; Huang, S. Novel approach to determine cathode two-phase-flow pressure drop of proton exchange membrane fuel cell and its application on water management. Appl. Energy 2017, 190, 713-724. [CrossRef]

11. Pei, P.; Li, Y.; Xu, H.; Wu, Z. A review on water fault diagnosis of PEMFC associated with the pressure drop. Appl. Energy 2016, 173, 366-385. [CrossRef]

12. Zhang, F.Y.; Yang, X.G.; Wang, C.Y. Liquid water removal from a polymer electrolyte fuel cell. J. Electrochem. Soc. 2006, 153, A225-A232. [CrossRef]

13. Chen, K.S.; Hickner, M.A.; Noble, D.R. Simplified models for predicting the onset of liquid water droplet instability at the gas diffusion layer/gas flow channel interface. Int. J. Energy Res. 2005, 29, 1113-1132. [CrossRef]

14. Kumbur, E.C.; Sharp, K.V.; Mench, M.M. Liquid droplet behavior and instability in a polymer electrolyte fuel cell flow channel. J. Power Sources 2006, 161, 333-345. [CrossRef]

15. Theodorakakos, A.; Ous, T.; Gavaises, M.; Nouri, J.M.; Nikolopoulos, N.; Yanagihara, H. Dynamics of water droplets detached from porous surfaces of relevance to PEM fuel cells. J. Colloid Interface Sci. 2006, 300, 673-687. [CrossRef] [PubMed]

16. Xie, J.; Xu, J.; Shang, W.; Zhang, K. Mode selection between sliding and rolling for droplet on inclined surface: Effect of surface wettability. Int. J. Heat Mass Transf. 2018, 122, 45-58. [CrossRef]

17. Quan, P.; Zhou, B.; Sobiesiak, A.; Liu, Z. Water behavior in serpentine microchannel for proton exchange membrane fuel cell cathode. J. Power Sources 2005, 152, 131-145. [CrossRef]

18. Jiao, K.; Zhou, B.; Quan, P. Liquid water transport in parallel serpentine channels with manifolds on cathode side of a PEM fuel cell stack. J. Power Sources 2006, 154, 124-137. [CrossRef]

19. Zhu, X.; Liao, Q.; Sun, P.C.; Djilali, N. Numerical investigation of water droplet dynamics in a low-temperature fuel cell microchannel: Effect of channel geometry. J. Power Sources 2010, 196, 801-812. [CrossRef]

20. Park, J.W.; Jiao, K.; Li, X. Numerical investigations on liquid water removal from the porous gas diffusion layer by reactant flow. Appl. Energy 2010, 87, 2180-2186. [CrossRef]

21. Yin, Y.; Wu, T.; He, P.; Du, Q.; Jiao, K. Numerical simulation of two-phase cross flow in microstructure of gas diffusion layer with variable contact angle. Int. J. Hydrogen Energy 2014, 39, 15772-15785. [CrossRef]

22. Cai, Y.H.; Hu, J.; Ma, H.P.; Yi, B.L.; Zhang, H.M. Effects of hydrophilic/hydrophobic properties on the water behavior in the micro-channels of a proton exchange membrane fuel cell. J. Power Sources 2006, 161, 843-848. [CrossRef]

23. Qin, Y.; Du, Q.; Yin, Y.; Jiao, K.; Li, X. Numerical investigation of water dynamics in a novel proton exchange membrane fuel cell flow channel. J. Power Sources 2013, 222, 150-160. [CrossRef]

24. Qin, Y.; Li, X.; Du, Q.; Yin, Y.; Jiao, K. Effect of wettability on water removal from the gas diffusion layer surface in a novel proton exchange membrane fuel cell flow channel. Int. J. Hydrogen Energy 2013, 38, 12879-12885. [CrossRef]

25. Qin, Y.; Li, X.; Jiao, K.; Du, Q.; Yin, Y. Effective removal and transport of water in a PEM fuel cell flow channel having a hydrophilic plate. Appl. Energy 2014, 113, 116-126. [CrossRef] 
26. Kim, H.Y.; Lee, H.J.; Kang, B.H. Sliding of liquid drops down an inclined solid surface. J. Colloid Interface Sci. 2002, 247, 372-380. [CrossRef] [PubMed]

27. Jiao, K.; Li, X. Effect of surface dynamic wettability in proton exchange membrane fuel cells. Int. J. Hydrogen Energy 2010, 35, 9095-9103. [CrossRef]

28. Chen, L.; He, Y.L.; Tao, W.Q. Effects of surface microstructures of gas diffusion layer on water droplet dynamic behaviors in a micro gas channel of proton exchange membrane fuel cells. Int. J. Heat Mass Transf. 2013, 60, 252-262. [CrossRef]

29. Das, P.K.; Grippin, A.; Kwong, A.; Weber, A.Z. Liquid-water-droplet adhesion-force measurements on fresh and aged fuel-cell gas-diffusion layers. J. Electrochem. Soc. 2012, 159, B489-B497. [CrossRef]

30. ANDYD. ANSYS FLUENT 12.1 User's Guide; ANSYS Inc.: Canonsburg, PA, USA, 2009.

31. Brackbill, J.U.; Kothe, D.B.; Zemach, C. A continuum method for modeling surface tension. J. Comp. Phys. 1992, 100, 335-354. [CrossRef]

32. Shakhshir, S.A.; Wang, Y.; Alaefour, I.; Li, X. The influence of channel wettability on two-phase flow and polymer electrolyte membrane fuel cell performance. ECS Trans. 2012, 42, 109-115.

33. Lv, C.; Yang, C.; Hao, P.; He, F.; Zheng, Q. Sliding of water droplets on microstructured hydrophobic surfaces. Langmuir 2010, 26, 8704-8708. [CrossRef] [PubMed]

34. Miwa, M.; Nakajima, A.; Fujishima, A.; Hashimoto, K.; Watanabe, T. Effects of the surface roughness on sliding angles of water droplets on superhydrophobic surfaces. Langmuir 2000, 16, 5754-5760. [CrossRef]

(C) 2018 by the authors. Licensee MDPI, Basel, Switzerland. This article is an open access article distributed under the terms and conditions of the Creative Commons Attribution (CC BY) license (http://creativecommons.org/licenses/by/4.0/). 